\title{
A Review of Graph-based Dependency Parsing
}

\author{
NanFang Jian \\ Zhengzhou Institute of Information Science and Technology, Henan 450001, China;
}

AndyNF2150@163.com

Keywords: Graph-based dependency parsing, Deep neural network, Recurrent neural network, Word embedding.

\begin{abstract}
Dependency parsing has been the mainstream of Syntactic parsing in NLP community. With the rapid development of deep neural network, more researches tend to utilize the neural network for dependency parsing, all these efforts has improved the accuracy of dependency parsing greatly. In this paper, we first introduce the theory of dependency parsing briefly. Then the main process of dependency parsing is analyzed in detail, including feature learning, training and decoding process. Lastly, we summarize the current problems and challenges during dependency parsing research, as well as discussing the further development.
\end{abstract}

\section{Introduction}

Natural Language Processing (NLP) is the key approach for computers to understand natural languages, which has been studied and applied for many years and always appearing people's attention. Intuitively, the process of Natural Language Processing is divided into three main layers: The first layer is called lexical analysis, which consists of word segmentation, Part-of-speech (POS) Tagging and Named Entity Recognition (NER), lexical analysis is considered as the basis of the later tasks. The second layer is called syntactic parsing, which is aimed to constructing a syntactic tree as the parsing result according to syntactic rules, and thus capture the correlations and modify relation of words in the sentence. Syntactic parsing has two main approaches, phrase structure parsing and dependency parsing, and dependency parsing is appealing more people to research now. The third layer is semantic parsing. This part aims at exploring and knowing the real meaning of sentence on the basis of syntactic parsing result. Currently, Semantic Role Labeling (SRL) is the main approach of semantic parsing, which has the best performance among all the parsing methods. Being the key process of Natural Language Processing, the performance of syntactic parsing has direct influence on the later semantic parsing, thus becoming the main research of Natural Language Processing.

Syntactic parsing is developed on the basis of corresponding syntactic theory. Among all the syntactic theories, dependency theory, one of the most popular theories, has attracted a lot of researchers due to its simple in form, easy to annotate and apply into practice. Dependency parsing aims to analyze a sentence according to the dependency rules and transforms the sentence into a dependency tree for the follow processing. Given a sentence $\mathrm{x}=\mathrm{x}_{1} \mathrm{x}_{2} \ldots \mathrm{x}_{\mathrm{n}}$ (xi denoting the $i$ th word of the sentence), the dependency tree could be formed as formula:

$$
d=\{(h, m, l) \mid 0 \leq h \leq n, 1 \leq m \leq n, l \in L\}
$$

Where $d$ could be represented as the dependency tree, $h$ denotes the head of the sentence as well as the father node in the dependency tree, similarly, $m$ represents the modifier of the sentence as well as the child in the dependency tree, $L$ represents a set of all dependency arcs which the arc $l$ belongs to. For example, a dependency arc $(h, m, l)$ denotes a dependency relationship from the head $w_{h}$ to modifier $w_{m}$, the type of the arc represents the dependency relations between two words in the sentence.

According to above theory, we could make dependency parsing for a sentence "Curry now plays for Warriors", all the structure and Part-of-speech follows the annotating standard of Chinese Penn 
Treebank 5 (CTB5). Figure 1 is the parsing result, where $w_{0}$ is a pseudo-word which appoint the head of a sentence, the arcs $(4,2$, SUB) represents "Curry" modifies "plays for", and the corresponding dependency type is SUB.

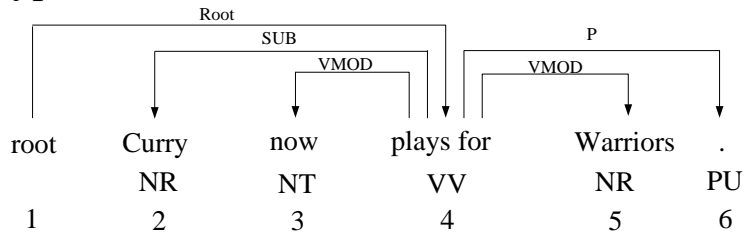

Fig. 1 An example of dependency tree

\section{Graph-based Dependency Parsing}

Currently, the two mainstream approaches of dependency parsing are graph-based dependency parsing and transition-based dependency parsing. During these five years, with the rapid development of deep neural network theory and application, an increasing number of researchers exploited this machine learning method to further improving the performance of dependency parsing, which significantly contributes to the combination of dependency parsing and deep neural network.

\subsection{Graph-based Dependency Parsing}

Graph-based dependency parsing approach was firstly proposed by McDonald (2005). Typical graph-based dependency parsing factors the dependency tree into directed sub-graph $G=(V, E)$ according to dependency theory, where $G$ denotes a set of vertexes of the sub-graph and $E$ denotes a set of edges of the sub-graph. Correspondingly, the vertexes in the sub-graph represent a sequence of words in the sentence and edges in the sub-graph represent the dependency relations between two words. Thus, the construction of the dependency tree could be transformed into a searching problem in the sub-graph using dynamic programming algorithm, and the searching aim is to find a sub-graph which owes the highest score. In order to prevent the complexity of the sub-graph and ensure a high searching speed, researchers attach some strong hypothesis to the sub-graph model. The first model is a simple first-order(the order represents the number of arcs in the most complex subtree) model that uses only dependent arcs in the sub-graph, and researchers then weaken the hypothesis by attaching higher order feature into the sub-graph, and thus improve the accuracy of model dependency.

Considering that a dependency tree is constructed for a sentence, which contains only projective subtree while other arcs out of projective tree is independent with each other, under this hypothesis, the score for the whole dependency tree could be could counted as the sum of all the score of the subtree, as follows:

$$
\operatorname{Score}(x, d)=w \cdot f(x, d)=\sum_{p \subseteq d} \text { Score }_{\text {subtree }}(x, p)
$$

Where $p$ represents a allowed subtree which contains one or more arcs in the dependency tree $d$, Score $_{\text {subree }}(x, p)$ denotes the score of this subtree, $f(x, d)$ denotes the feature embedding of $(x, d)$ and $w$ denotes the weight embedding of the corresponding feature embedding.

\subsection{Neural Network Based Dependency Parsing}

In recent years, a lot of researches were conducted on natural language syntactic parsing based on deep learning. Therefore, many current researches focus on dependency analysis model under the neural network. The template is used to format your paper and style the text.

\section{Feed-forward network dependency parsing model}

Feed-forward neural network (FNN) is a simple neural network model, Inside FNN, parameter value performs a one-way transmission from input layer to output layer, the parameter was renewed by the gradient which was derived by standard back-propagation algorithm. Chen and Manning firstly raised a Dependency parsing model in 2014 which was based on FNN inside transfer. By 
using similar method, Pei raised a solution of Graph-based dependency parsing model by FNN. This Dependency parsing model shows in figure 2, it has three major features listed below:

(1) Its core feature is that its vector use word embedding and POS-tag embedding compared with previous non-neural network Dependency parsing model, its feature engineering work greatly reduced.

(2) Phrase embedding is calculated by the hidden cell vector of FNN (See the right of Fig.1), the phrase embedding is added to the FNN input layer as additional feature. Because the hidden layer of FNN was calculated by feature combination, it contains more contextual information.

(3) A unique hidden layer activation function was used: $g(t)=\tanh \left(x^{3}+x\right)$

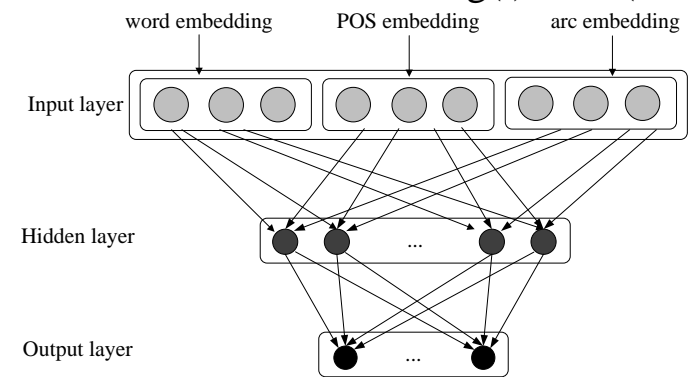

Fig. 2 Feed-forward neural network graph-based dependency parsing model

\section{LSTM dependency paring model}

As a variant of recurrent neural network, the long short-term memory (LSTM) was designed to solve the gradient vanishing problem. LSTM includes one "memory cell" and three "control gates" which are used to control when to "memorize" and to "forget". Generally, LSTM has one input gate, one forget gate and one output gate. The input gate determine the recorded rate that current moment input is allowed to enter into memory cells. The forget gate determine the forgotten rate of current moment input.

At the moment of $t$, given current input vector $x_{t}$ previous moment hidden condition $h_{t-1}$, LSTM cell can calculate current moment hidden condition $h_{t}$ by its own inside circulation and renew.

$$
\begin{aligned}
& \boldsymbol{i}_{\boldsymbol{t}}=\sigma\left(\boldsymbol{U}^{i} \boldsymbol{x}_{\boldsymbol{t}}+\boldsymbol{W}^{\boldsymbol{i}} \boldsymbol{h}_{\boldsymbol{t}-1}+\boldsymbol{b}^{\boldsymbol{i}}\right) \\
& \boldsymbol{f}_{\boldsymbol{t}}=\sigma\left(\boldsymbol{U}^{f} \boldsymbol{x}_{\boldsymbol{t}}+\boldsymbol{W}^{f} \boldsymbol{h}_{\boldsymbol{t}-1}+\boldsymbol{b}^{f}\right) \\
& \boldsymbol{o}_{t}=\sigma\left(\boldsymbol{U}^{o} \boldsymbol{x}_{t}+\boldsymbol{W}^{o} \boldsymbol{h}_{t-1}+\boldsymbol{b}^{o}\right) \\
& \boldsymbol{c}_{\boldsymbol{t}}=\boldsymbol{f}_{\boldsymbol{t}} \odot \boldsymbol{c}_{\boldsymbol{t}-1}+\boldsymbol{i}_{\boldsymbol{t}} \odot \varphi\left(\boldsymbol{U}^{c} \boldsymbol{x}_{\boldsymbol{t}}+\boldsymbol{W}^{c} \boldsymbol{h}_{t-1}+\boldsymbol{b}^{c}\right) \\
& \boldsymbol{h}_{\boldsymbol{t}}=\boldsymbol{o}_{\boldsymbol{t}} \odot \varphi\left(\boldsymbol{c}_{\boldsymbol{t}}\right)
\end{aligned}
$$

Where $x_{t}$ is the memory cell input at moment $t . U^{i}, U^{f}, U^{o}, U^{c}, W^{i}, W^{f}, W^{o}, W^{c}$ are the parameter arrays, and $b^{i}, b^{f}, b^{o}, b^{c}$ are the corresponding vectors.

Previous work has proved that long Short-Term Memory (LSTM) network is more efficient than simple recurrent neural network (RNN). Similar to Feed-forward neural network (FNN), BPTT (back propagation through time) was used to seek the network parameter partial conductance of the loss function of the LSNN(long short-term neural network), meanwhile, gradient descent methods was used for network parameter learning. 


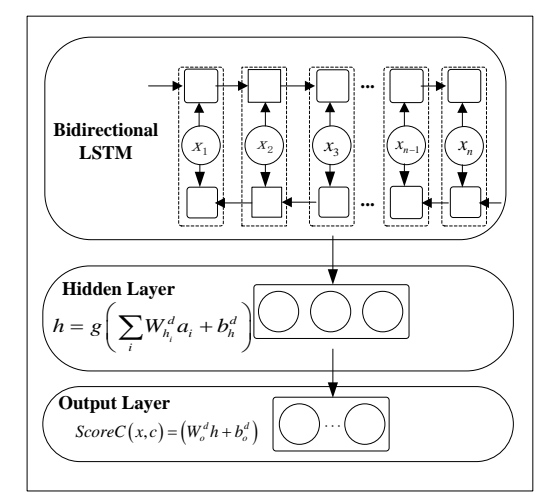

Fig. 3 LSTM neural network graph-based dependency paring model

In 2016, by using BiLSTM, Wang conducted graph-based dependency parsing (Fig. 3), its comparison model was the FNN parsing analysis model raised by Pei. Compared with the FNN parsing analysis model, Wang's model has some better features:

On the one hand, different from FNN, input word vector was handled sequentially by LSTM, this method can learn longer distance dependency relationship feature. On the other hand, different from phrase embedding, the hidden cell of the forward LSTM was used to calculate the segment embedding. The extracted segment embedding feature contains more information than common word vector, so that it got better performance at long-distance dependency parsing, although these two methods share similar philosophy on vector features calculation.

\section{Main Process of Graph-based Dependency Parsing}

\subsection{Feature Learning}

Feature embedding plays an important role in syntactic parsing which is used to describe the features of the specific language and context related features, thus we could compute the score of all the dependency subtrees. Among the basic graph-based dependency parsin, most features using in syntactic parsing comes from domain specialist, this feature is called hand-crafted features and is captured from feature template.

Due to the complex characters of natural language, syntactic parsing needs a mass of meaning features. Conventionally these features was designed and captured by researchers. This leads to heavy relying on an enormous number of hand-crafted features. However, these hand-drafted features could capture inherent correlations of words or sentences. Moreover, these features, especially lexicalized features are highly sparse, and feature templates are also incomplete, therefore, researchers attempt to seek approaches which could extract useful features automatically. In recent years, many researches use deep neural network model to learn dense features of words, POS tagging and dependency arcs, these models have obtained pretty good performance compared with conventional approaches and achieved a higher parsing speed.

\subsection{Training}

As for dependency parsing models, training algorithm is to learn model arguments and feature embedding by process a large number of labeled data or unlabeled data. The effectiveness of arguments learned by training algorithm have important influence on parsing result, it is widely acknowledged that better arguments will contribute more to accuracy of dependency parsing models.

Commonly, graph-based dependency parsing utilizes training algorithms averaged perception (AP), passive aggressive (PA), averaged margin infused relaxed (MIRA), these training algorithms are called online learning algorithm. Totally, online learning algorithm will input a row of training data to acquire parsing result within weight embedding $w^{(k)}$, and give back-prorogation and update weight embedding $w(k+1)$ by compare the parsing result with correct result. After several iterations, the final weight embedding will be the best arguments for dependency parsing model.

For the neural dependency paring model, training algorithms for deep neural network, such as cross entropy training and average perceptron (AP) training, are usually used to define the object 
function. After calculating the gradient for each parameter of the neural network model, the back propagation algorithm will be adopted to update the model parameter, until the network is well trained.

\subsection{Decoding}

Graph-based dependency parsing aims to search a dependency subtree in the directed graph which obtains the highest score. Correspondingly, decoding algorithms adopt dynamic programming approach, these algorithm combine the results of sibling words and finally obtain result of the whole sentence. The first-order graph-based dependency model uses Eisner algorithm which ensures parsing result to be a projective tree. The second-order and third-order graph-based model expand Eisner algorithm and consider the score of adjacent siblings and head-modifier-grandchild, these higher order models attach more features thus achieves a better accuracy.

\section{Discussion}

Graph-based dependency parsing is described with neural networks in the recent years, these researches include FNN, RNN and LSTM. Among these researches, LSTM model achieves better performance than others. Additionally, in the most recent researches, researchers attempt to conduct dependency parsing using attention-based memory network, in this way, the process of the Dependency parsing was converted to the process of dynamical selection of each word head node, its final purpose was also the same, that is to build the dependency graph which corresponds to the sentence, here maximum spanning tree algorithm is used for decoding. The method was not introduced in this article.

Due to that the accuracy rate of Dependency parsing is pretty high using single model, future research might focus on combining parsing analysis work and other works together, such as POS tagging and semantic analysis etc. There is some interaction relationship features along these jobs, so that they can supply auxiliary information to each other. Additionally, current Dependency parsing is only based on single language, thus it will be an important research to conduct multilingual parsing in the future.

\section{Conclusion}

This paper systematically concludes the graph-based dependency parsing theoretically and practically. We first introduced the graph-based dependency parsing in detail, including the traditional graph-based models and the recent neural graph-based dependency parsing model. Then we analyzed feature learning, training algorithms and decoding algorithms for dependency parsing. Lastly, we discussed the current problems and challenges. To conclude, this paper could contribute to a better understanding of dependency parsing for researchers in related fields.

\section{References}

[1] McDonald R, Crammer K, Pereira F. Online Large-Margin Training of Dependency Parsers[C]. Proceedings of ACL 2005. 2005:91-98.

[2] McDonald R, Pereira F. Online Learning of Approximate Dependency Parsing Algorithms[C]. Proceedings of EACL 2006. 2006.

[3] Carreras X. Experiments with a Higher-Order Projective Dependency Parser[C]. Proceedings of EMNLP/CoNLL. 2007:141-150.

[4] Koo T, Collins M. Efficient Third-Order Dependency Parsers[C]. Proceedings of the 48th Annual Meeting of the Association for Computational Linguistics. 2010:1-11.

[5] Eisner J. Bilexical Grammars and a Cubic-Time Probabilistic Parser[C]. Proceedings of the 5th International Workshop on Parsing Technologies (IWPT). 1997:54-65. 
[6] Eisner J. Bilexical Grammars and Their Cubic-Time Parsing Algorithms[M]. Bunt H C, Nijholt A. Advances in Probabilistic and Other Parsing Technologies. Kluwer Academic Publishers, 2000:29-62.

[7] Collins M. Discriminative Training Methods for Hidden Markov Models: Theory and Experiments with Perceptron Algorithms[C]. Proceedings of EMNLP 2002. 2002.

[8] Zhenghua Li, Wanxiang Che, Ting Liu. Improving Chinese POS Tagging with Dependency Parsing. In the Proceedings of the 5th International Joint Conference on Natural Language Processing (IJCNLP 2011). 2011.08, pp. 1447-1451, Chiang Mai, Thailand.

[9] Danqi Chen and Christopher Manning. 2014. A fast and accurate dependency parser using neural networks. In Proceedings of the 2014 Conference on Empirical Methods in Natural Language Processing(EMNLP), pages 740-750, Doha, Qatar, October. Association for Computational Linguistics.

[10]Phong Le and Willem Zuidema. 2014. The insideoutside recursive neural network model for dependency parsing. In Proceedings of the 2014 Conference on Empirical Methods in Natural Language Processing (EMNLP), pages 729-739, Doha, Qatar, October. Association for Computational Linguistics.

[11]Eliyahu Kiperwasser and Yoav Goldberg. ACL 2016. Simple and accurate dependency parsing using bidirectional lstm feature representations. Transactions of the Association for Computational Linguistics 4:313-327.

[12]Wang, Wenhui, and B. Chang. Graph-based Dependency Parsing with Bidirectional LSTM. Meeting of the Association for Computational Linguistics 2016.

[13]Hao Cheng, Hao Fang, Xiaodong He, Jianfeng Gao, and Li Deng. 2016. Bi-directional attention with agreement for dependency parsing. In Proceedings of EMNLP 2016. Austin, Texas, pages 2204-2214.

[14]Dyer, Chris, et al. Transition-Based Dependency Parsing with Stack Long Short-Term Memory. Computer Science 2015. 